\title{
FM 9:
}

Highlights in the Exploration of Small Worlds 


\title{
Comet composition and Lab
}

\author{
Dominique Bockelée-Morvan \\ LESIA, Observatoire de Paris, LESIA/CNRS, UPMC, Université Paris-Diderot, \\ F-92195 Meudon, France \\ email: dominique.bockelee@obspm.fr
}

The XXIX IAU General Assembly took place during the golden year of the exploration of small solar system bodies. With the Rosetta ESA mission around comet 67P, NASA Dawn and New Horizons missions nearby dwarf planets Ceres and Pluto, respectively, and the NASA/Cassini mission in Saturn neighborhood, year 2015 marked an important step towards further understanding of small solar system bodies. On August 11-13, Focus meeting 9 "Highlights in the exploration of small worlds" gathered scientists of all over the world to present and discuss the spectacular results obtained from these missions, as well as recent achievements obtained from past missions, comprehensive spectroscopic surveys from space (e.g., Herschel, NEOWISE, Gaia), ground-based observations, and geochemical analyses. This meeting was also the opportunity to discuss the state of our understanding of the nature of the various populations of small bodies in the Solar System, including icy satellites, in a cosmo-chemistry perspective.

Investigating in detail the physical and chemical properties of asteroids, comets, transneptunian objects and dwarf planets is indeed of tremendous importance for understanding the formation of the Solar System, and overall the process of star and planet formation. These bodies are the remnants - either fragments or "survivors" - of the swarm of planetesimals from which the planets were formed. They are thus primitive leftover building blocks of the Solar System formation process that can offer clues to the chemical mixture from which the planets formed some 4.6 billion years ago.

It is now clear that there was a general mixing of the different populations of small bodies at the earlier stages of the Solar System, as a result of planetary migration. Indeed, geochemical evidence obtained from analyses of extraterrestrial samples and the recent discovery of active and icy asteroids, show that differences between primitive asteroids and comets, are much less sharp than previously thought. During this Focus Meeting, the interrelationships between the various populations of small bodies were investigated through a detailed comparison of their physical and chemical properties, which is an mandatory step towards understanding their formation conditions and their evolutionary paths.

The interpretation of the isotopic, molecular and mineralogical properties of primitive solar system material is complex, and can only be achieved through a multi-disciplinary approach. A full session of Focus Meeting 9 discussed on solar system formation in the light of proto-planetary disk models, experimental works and recent insights on the composition of comets and proto-planetary disks.

Dominique Bockelée-Morvan, Daniel Hestroffer, Paola Caselli Chairs

Michael A'Hearn, Elisabetta Dotto, Ian Franchi, Karen Meech, Hideyo Kawakita, Diane Wooden, Jorge M. Carvano, Javier Licandro, Gabriel Tobie, Richard Binzel, Mikael Granvik, Wing Ip, Steven Charnley 